\title{
Immune-related adverse events associated with immune checkpoint inhibitors: a call to action for collecting and sharing clinical trial and real-world data
}

To cite: Reynolds KL, Arora S, Elayavilli RK, et al. Immunerelated adverse events associated with immune checkpoint inhibitors: a call to action for collecting and sharing clinical trial and real-world data. Journal for ImmunoTherapy of Cancer 2021;9:e002896. doi:10.1136/jitc-2021-002896

Accepted 30 May 2021

Check for updates

(c) Author(s) (or their employer(s)) 2021. Re-use permitted under CC BY-NC. No commercial re-use. See rights and permissions. Published by BMJ.

For numbered affiliations see end of article.

Correspondence to

Dr Kerry L Reynolds;

kreynolds7@partners.org

\section{ABSTRACT}

Immune checkpoint inhibitors (ICls) have revolutionized the treatment of cancer, improving outcomes in patients with advanced malignancies. The use of ICls in clinical practice, and the number of $\mathrm{ICI}$ clinical trials, are rapidly increasing. The use of ICls in combination with other forms of cancer therapy, such as chemotherapy, radiotherapy, or targeted therapy, is also expanding. However, immunerelated adverse events (irAEs) can be serious in up to a third of patients. Critical questions remain surrounding the characteristics and outcomes of irAEs, and how they may affect the overall risk-benefit relationship for combination therapies. This article proposes a framework for irAE classification and reporting, and identifies limitations in the capture and sharing of data on irAEs from current clinical trial and real-world data. We outline key gaps and suggestions for clinicians, clinical investigators, drug sponsors, patients, and other stakeholders to make these critical data more available to researchers for pooled analysis, to advance contemporary understanding of irAEs, and ultimately improve the efficacy of ICls.

\section{INTRODUCTION}

The development and approval of immune checkpoint inhibitors (ICIs) targeting cytotoxic T-lymphocyte-associated protein 4 (CTLA-4) and programmed cell death protein 1 (PD-1) and its ligand (PD-L1) have altered the treatment landscape for several different types of cancers (figure 1). ICIs provide benefit in terms of improvement in overall survival $^{1}$ and durable response rates for a variety of cancer patients, however the vast majority ${ }^{2}$ of patients who receive these therapies experience immune-related adverse events (irAEs). These ICI-related inflammatory toxicities are distinct from those associated with other systemic cancer therapies. ${ }^{3}$ The risk of an ICI affecting any organ is as high as $86 \%$ for a CTLA-4 inhibitor and $82 \%$ for anti-PD-1/PD-L1 agents. ${ }^{1}$ Oftentimes, the adverse events (AEs) are not clinically severe ${ }^{45}$ however, between $14 \%$ and $29 \%$ of patients experience Common Terminology Criteria for Adverse Events (CTCAE) grade 3 or 4 irAEs, depending on the ICI agent. ${ }^{6}$ In rare cases, multiple organs may be simultaneously affected ${ }^{78}$ (figure 2).

In an effort to extend the benefits of ICIs to a larger group of patients, there are efforts to design rational combination strategies with other ICIs or forms of cancer therapy, such as chemotherapy, radiotherapy, or targeted therapy. ${ }^{9}$ However, AEs are a diagnostic challenge in the context of ICI combination treatment. It is increasingly difficult to determine the underlying agent responsible for the toxicity, which also has important implications for management. By correctly phenotyping irAEs and understanding the varied presentations and natural history, we can begin to develop a framework to distinguish between AEs associated with conventional chemotherapy or targeted therapy and immunotherapy.

Although clinical trial and real-world data on characterization and management of irAEs are beginning to emerge ${ }^{10-12}$ both types of data have critical limitations and gaps that hinder the ability to aggregate the data and conduct pooled analyses. The challenges include lack of appropriate data sharing and lack of standardization in AE documentation in clinical trials and in real-world settings. Specific actions could be taken to overcome gaps so that available data can be meaningfully studied in aggregate, thus enabling more accurate, complete characterization of the irAE; identification of varying rates and types 


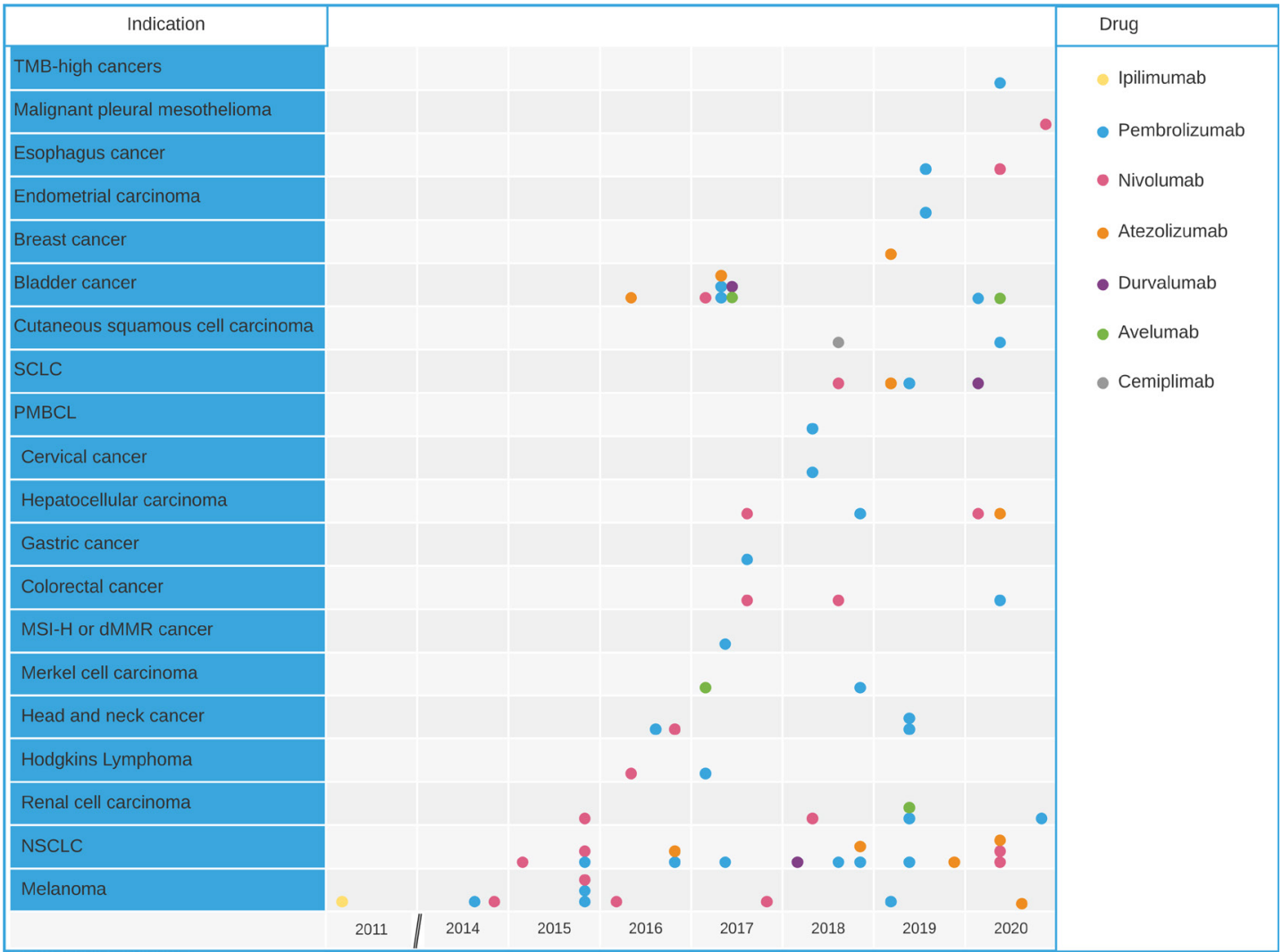

Figure 1 Timeline depicting the year and indications for which immune checkpoint inhibitors were granted approval by the US Food and Drug Administration. dMMR, deficient MisMatch Repair; MSI-H, high levels of microsatellite instability; NSCLC, non-small cell lung cancer; PMBCL, primary mediastinal large B-cell lymphoma; SCLC, small cell lung cancer; TMB, tumor mutational burden.

of irAEs with different ICI-drug combinations; and identification of rare but clinically significant irAEs. We are at a crucial time to improve the diagnosis and management of irAEs and all stakeholders, from clinicians, clinical investigators, drug sponsors, and patients, should work together to achieve these goals.

In support of the broad interest in and support for these efforts among various groups, a symposium was jointly convened by the US Food and Drug Administration (FDA) and Project Data Sphere, a not-for-profit initiative of the CEO Roundtable on Cancer,${ }^{13}$ on the topic of irAEs and developing more effective approaches to study irAEs, and was widely attended. Following this meeting, representatives from key groups, major academic medical centers, government agencies, non-profit organizations, and pharmaceutical and biotech companies, continued the discussion to identify areas where progress could lead to critical advances in the understanding of irAEs and ultimately improvements in ICI treatment. These areas, along with specific limitations and barriers and potential actions to overcome them, are the focus of this article: (1) the need to standardize AEs/irAEs in clinical trial reporting; (2) the need to improve reporting of realworld data; and (3) the actions that all stakeholders can collectively take to facilitate better reporting and understanding of AEs.

\section{IMPROVING CLINICAL TRIAL DATA REPORTING: STANDARDIZING AE TERMINOLOGY AND CASE REPORT FORMS WOULD FACILITATE POOLED ANALYSES OF TRIAL DATA Lessons from irAE clinical trial data}

Clinical trials examining the safety and efficacy of ICIs offer an excellent source of data for studying how irAEs may present differently as monotherapy or in combination with chemotherapy or other anti-cancer agents. In these rigorous environments, study participants are closely monitored with frequent follow-up visits, detailed symptom assessments, and careful documentation of laboratory and histopathological data so that even subtle AEs may be captured. By aggregating large numbers of patients from similar trials we can learn more about various aspects of irAEs, including risk factors for developing irAEs and important clinical manifestations, timing of onset, how time to treatment of toxicity impacts outcomes, time to resolution, percentage of refractory cases, and early recognition and intervention to reduce the severity and potential long-term morbidity caused by the irAE.

Various investigational projects, compiling ICI clinical trial data, are being performed by researchers in academia and regulatory agencies such as the US FDA to further understand the safety profile of ICIs. Over the past few years, the FDA has conducted several exploratory 
EXAMPLES OF ICI-INDUCED TOXICITIES ACROSS ORGAN SYSTEMS

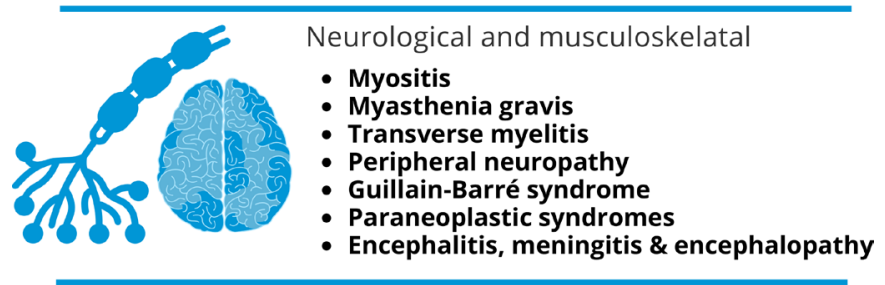

Endocrine

- Hypophysitis

- Thyroiditis

- Hypo/Hyper-thyroidism

- Primary adrenal insufficiency

- Type 1 diabetes mellitus

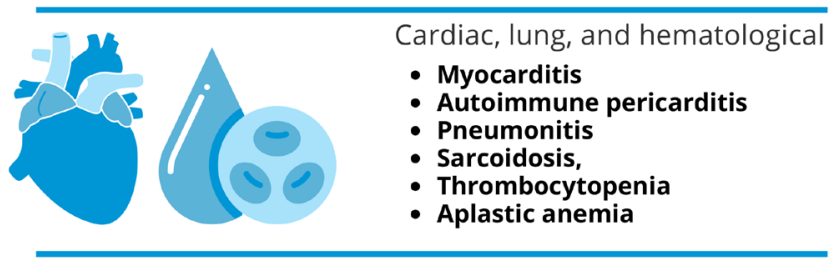

Dermatological

- Maculopapular rash

- Pruritis

- Psoriasiform eruptions

- Lichenoid eruptions

- Bullous pemphigoid

- Vitiligo
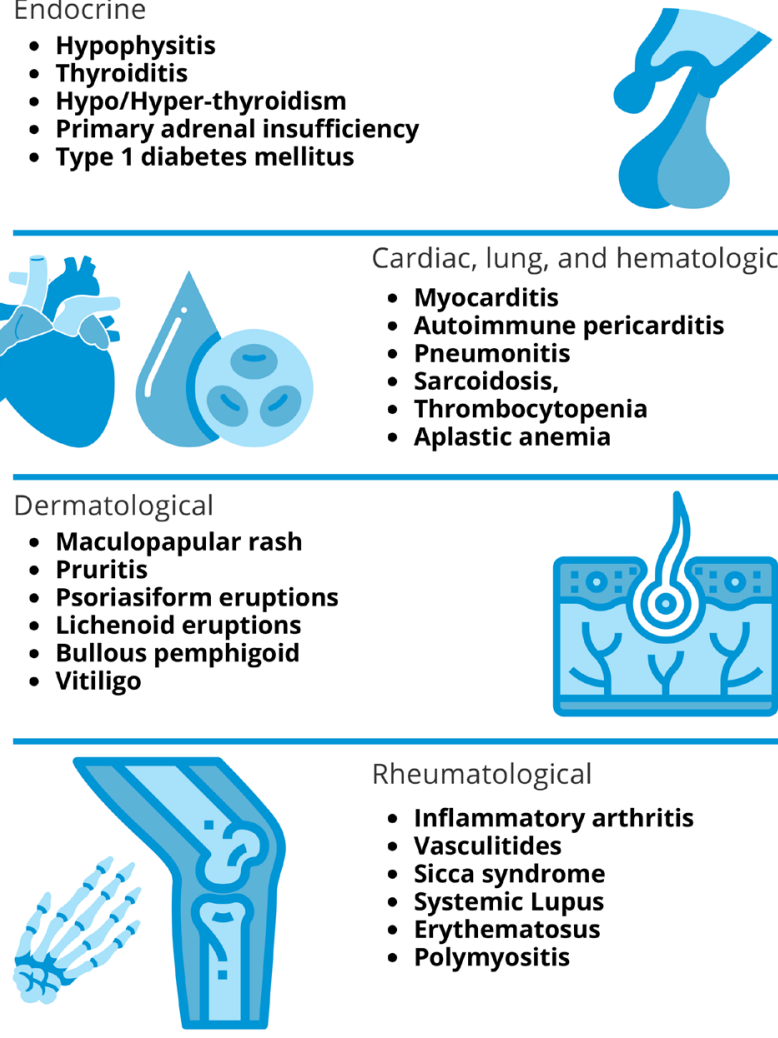

Rheumatological

- Inflammatory arthritis

- Vasculitides

- Sicca syndrome

- Systemic Lupus

- Erythematosus

- Polymyositis

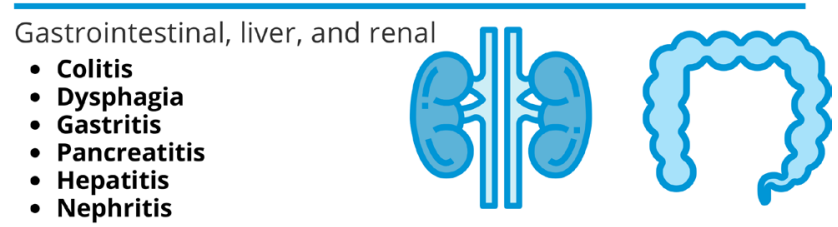

Figure 2 Overview of major immune-related adverse events that have been reported according to organ system.

analyses by aggregating data from several phases 2 and 3 ICI clinical trials (currently includes over 65 trials, spanning more than 25000 patients) submitted to the Agency as part of new or supplemental Biologics License Applications. One such study evaluated cardiovascular AEs and found higher rates of these AEs in the ICI therapy group than in the non-ICI therapy group, as well as higher rates of cardiovascular AEs in patients receiving ICI+ICI combination treatment versus ICI monotherapy. ${ }^{14}$

Academic research groups have performed analyses of published clinical trial data to compare the rates and severity of irAEs for different ICI agents across different cancer types. Wang et al performed a meta-analysis of 34 trials, which concluded that rates of colitis and diarrhea were higher among patients treated with a combination of ipilimumab and PD-1/PD-L1 inhibitor compared with any ICI monotherapy for a range of cancer types. ${ }^{10}$ A metanalysis of 48 trials revealed a positive correlation between irAEs, particularly those that affect the skin and gastrointestinal tract, and clinical response to nivolumab alone or in combination with ipilimumab. ${ }^{15}$ However, these analyses are limited by their inability to evaluate patient-level information. Additionally, academic groups have reviewed irAE cases in the setting of cancer care centers and, for example, identified genetic variations associated with risk of developing irAEs, such as the association between certain HLA haplotypes and onset of insulin-dependent diabetes in patients receiving a PD-1/ PD-L1 inhibitor. ${ }^{16}$

In addition to providing safety and efficacy data, clinical trials can directly contribute to translational studies that help advance understanding of the molecular pathogenesis and treatment of severe irAEs. Alliance A151804, which was launched by the National Clinical Trials Network Alliance for Clinical Trials in Oncology in January $2020,{ }^{17}$ is recruiting patients enrolled in National Cancer Institute (NCI) ICI trials who experience severe (grade 3 or 4 ) irAEs. The effort centers around collecting biospecimens such as tissue and blood samples from patients at the time they experience the $\mathrm{AE}$ and at several subsequent timepoints (1, 3, 6, and 12 months), which are annotated, stored in a central repository, and distributed to researchers. This translational effort has the potential to better define the mechanisms underlying the development of irAEs, which in turn may allow the field to identify biomarkers that predict those at highest risk and advance evidence-based treatment strategies to manage irAEs. Discovery of biomarkers associated with irAEs is an area of active investigation. Recent studies have also used data sources beyond clinical trial data, such as patient studies and pharmacovigilance data combined with omics data, to identify intestinal microbiome profiles, tumor immune biomarkers, and differential gene expression associated with elevated irAE risk. ${ }^{18-20}$

\section{Barriers to aggregating irAE clinical trial data}

Clinical investigators face several challenges related to nomenclature and definitions of toxicity in capturing the characteristics and severity of irAEs. In general, the goldstandard mechanism that can definitively diagnose irAEs, and thus confirm that the toxicity is due to ICI treatment, and not a combination treatment partner such as chemotherapy or an alternative diagnosis, is biopsy with histopathological evidence of inflammation. However, biopsy is not possible for all patients because of difficulty accessing the target tissue and safety concerns. While novel non-invasive diagnostic tests are actively being investigated, none can currently make a definitive diagnosis of irAEs. Although there are published guidelines available to diagnose irAEs, ${ }^{31-23}$ and criteria to assess severity, ${ }^{24}$ specific diagnostic criteria have not been established for many irAEs, and in these circumstances, clinicians 
must use their discretion in describing toxic events and attributing them to the ICI. The lack of comprehensive nomenclature for irAEs and standardized definitions and grading criteria furthermore preclude in-depth analyses of toxicities documented in published clinical trials. Although trials generally use CTCAE grading, this tool can present challenges because it was originally developed for chemotherapy-related AEs and is often not robust enough to cover certain irAEs. ${ }^{24}{ }^{25}$ Furthermore, because trials do not publish patient-level information, it is not possible to distinguish between similar symptoms which may present with varying degrees of severity, such as diarrhea and colitis or thyroiditis and hypothyroidism. Nor is it possible to definitively determine the frequency with which multiple toxicities occurred in the same patient or the trajectory and outcome of toxicities for individual patients.

Another barrier to aggregating AE data between ICI clinical trials arises from differences between reporting the specifics of workup and treatment for irAEs, including time to onset of the AE, start and stop dates of steroids, maximum dose and duration of treatment administered, need for reescalation or second-line immunomodulators, action taken with the ICI (dose reduced, interrupted, discontinued etc).

\section{Facilitating pooled analyses of irAE clinical trial data}

In order to improve characterization of the safety of ICIs, consensus should be developed on diagnostic workups for irAEs and irAE definitions, as well as standardized clinical trial reporting by a panel of expert clinicians, representatives from pharmaceutical and biotech companies, clinical research organizations (CROs), and other groups. In fact, there are already ongoing efforts to standardize irAE definitions and provide more clear decision support for diagnosing irAEs in certain organ systems, but these efforts will need to be coordinated and embraced broadly to be fully implemented.

The authors submit it would be feasible to establish a standardized core section, or minimum data set, of the case report forms (CRFs) used to collect patient safety data in clinical trials because of the fact that anticipated AEs are similar across many ICIs. Such a minimum data set would include fields to indicate: specific irAE symptoms; date of irAE resolution; duration of steroid use; need to re-escalate steroids; need to use second-line immunosuppression, such as infliximab, mycophenolate mofetil, and abatacept; need for hospitalization; need for ICU level care; occurrence of the same or new irAE if patient was rechallenged; presence of multiple toxicities and list of the organs involved, and start and resolution dates. Many of these fields are not commonly included in CRFs, and importantly, would allow investigation of the trajectory, duration, and outcome of AEs.

The minimum data set would not be all encompassing, and it would be important to ensure drug sponsors have the ability to design or modify CRFs differently for diverse clinical trials. For example, a drug sponsor may anticipate a unique AE profile owing to the mechanism of action of the drug, particularly drugs in development that target novel immune checkpoints, ${ }^{26}$ and decide to add CRF fields to monitor for these specific AEs.

In addition to standardizing data collection in clinical trials, the use of an irAE minimum data set would help standardize reporting and preparation of CRFs for submission of trial data to regulatory agencies for drug approval. Data managers and CRO representatives often use electronic health records (EHRs) to complete CRFs and there could be opportunities to integrate the minimum data set into these systems, streamlining the workflow. Beyond the benefits discussed thus far, a standardized CRF section could also improve the study of other aspects of ICI therapy and design of future clinical trials of ICIs, as well as help inform the language of drug labels.

\section{IMPROVING REAL-WORLD DATA: EXPANDING AE REPORTING TO CAPTURE RARE EVENTS AND PROMPT FURTHER STUDY} Contributions of real-world data for understanding irAEs

For ICIs that are used in clinical practice, pharmacovigilance databases collect real-world post-marketing safety data on irAEs, and allow for the continued investigation of their characteristics and outcomes in a manner that is complementary to clinical trials. ${ }^{27}$ The World Health Organization (WHO) VigiBase and FDA Adverse Event Reporting System (FAERS) are the largest international pharmacovigilance databases and considered the gold standards for the industry for postmarketing surveillance. Whereas VigiBase is accessible to health professionals, FAERS is a publicly available and searchable database to which anyone can voluntarily report AEs that occur in patients taking drugs for both FDA-approved indications and off-label through the MedWatch online reporting system. ${ }^{28}$ Despite their limitations, which we discuss in the subsequent section, these data sets have important strengths, such as the fact that reports come from a patient population that is larger and more diverse than typical clinical trial participant populations, and unlike clinical trials, toxicities can be documented outside of a defined trial follow-up period. For many drugs, FAERS data have led to evaluation of safety signals and the addition of safety information to the drug labels. ${ }^{29}$

There has been growing interest in developing statistical methods to analyze FAERS, VigiBase, and other large pharmacovigilance databases in order to capture AEs that may not have been detected in clinical trials and also to corroborate the types of AEs documented in clinical trials. Although these types of data sets cannot be used to determine the incidence of AEs, due to the nonroutine, voluntary nature of reporting and the potential for duplicate reports, analyses have been conducted to glean whether certain toxicities are likely associated with ICI use based on disproportionalities in the database between ICI reports and reports for all other drugs, or reports for other cancer therapies. ${ }^{27}$ One such study used 
the FAERS database to detect signals of ICI-associated AEs and found higher reporting odds ratios for AEs associated with ICI +ICI combination therapy as compared with ICI monotherapy, which corroborates clinical observations. ${ }^{30}$ A study of VigiBase found that ICIs were associated with higher incidence of reports of certain classes of neurologic AEs, including neuromuscular junction dysfunction and cerebral artery vasculitis, compared with the overall incidence of these reports in the full data set. ${ }^{31}$ In other types of studies, the FAERS database has been analyzed to explore, for example, age-based differences in various irAEs. ${ }^{32}$ The additional types of information that can be gleaned from FAERS and its potential to support clinical trial and other types of data makes its open access critical.

Another opportunity afforded by the use of real-world data sets is the study of rare and vulnerable patient populations that would typically not be well represented in clinical trials. As one example, Flatiron Health and the NCI analyzed data in the Flatiron database, which has been developed using EHRs from melanoma patients with pre-existing liver or kidney dysfunction who were treated with ICIs. ${ }^{33}$ While patients with baseline organ dysfunction might be excluded from a clinical trial for legitimate reasons, once a drug is approved, clinicians are forced to make treatment decisions in the best interests of their patients, while also taking into account pre-existing comorbidities. Real-world data can help fill in knowledge gaps such as these, informing both patients and their clinicians of the safety and tolerability of novel regimens that were never formally studied in patients with their unique features, enabling risk-benefit assessments prior to an intervention with an ICI.

\section{Limitations of real-world data sets}

Any individual, including physicians, pharmacists, nurses, patients, and family members, can voluntarily submit reports directly to FAERS. However, the vast majority of reports submitted to FAERS (about 95\%) come from drug sponsors, which are required by law to submit all $\mathrm{AE}$ reports to the FDA. ${ }^{34}$ The fact that the majority of FAERS reports come from drug sponsors indicates that healthcare providers are not routinely submitting AE directly to FAERS. Moreover, because of the largely voluntary nature of reporting, AEs may not be reported at all; and even when they are reported, the individual completing the form may provide only limited information and may omit important information such as the patient's relevant medical history, concomitant drug use, dates the $\mathrm{AE}$ was experienced, testing done to diagnose the $\mathrm{AE}$, and treatment administered.

\section{Approaches to overcome limitations of real-world data sets}

Given the numerous ways that real-world data improve understanding of irAEs, it is important to increase awareness among healthcare providers about the importance of reporting toxicities experienced by their patients being treated with ICIs. Many cancer centers within academic medical institutions, including Massachusetts General
Hospital, Johns Hopkins University, and Vanderbilt University Medical Center, now have toxicity services made up of immuno-oncology experts at the institution that monitor and study immunotherapy side effects in patients. ${ }^{25}$ It should become standard practice at these services to report serious and unexpected AEs to FAERS, or to the drug manufacturer who could then report the event to FAERS. However, meaningful strides in toxicity reporting will require an investment at all cancer care centers in experienced staff dedicated to the timeintensive process of evaluating patients and documenting their symptoms. Alternatively, it may be possible in the longer term to increase toxicity reporting through the development of technological infrastructure. Ideally, to streamline this effort, and make $\mathrm{AE}$ reporting reliable and practical, it should be integrated into the workflow at the service and incorporated into software at cancer centers for AE reporting, similar to the tools used by drug sponsors to submit AE information they receive to FAERS.

In a recent study, researchers developed a pharmacovigilance signal detection framework to detect irAEs. This study demonstrated the feasibility of creating a scalable model, integrating spontaneous reporting (FAERS) and EHR data sets. The platform is now open source and publicly available and provides a tool that other researchers could use with their own data sets to improve the capture of real-world irAE data. ${ }^{35}$

\section{SECTION 4: CALL TO ACTION: ALL STAKEHOLDERS PLAY A ROLE IN THE PROCESS}

We identify five key actionable steps that could greatly advance our understanding of irAEs, and ultimately improve treatment and prevention: (1) standardize irAE definitions; (2) standardize data requirements, such as through the use of a minimum data set in clinical trial CRFs, for submission of new drug applications to regulatory bodies; (3) ensure real-world AE data are routinely reported in a timely and thorough fashion by care teams; (4) develop and adopt technology to facilitate the consistent reporting of real-world data by patients and providers; (5) make data readily accessible in order to fuel discovery.

The burden of responding to the calls to action suggested herein regarding ICIs would fall on multiple stakeholders. The responsibility of standardizing irAE definitions and reporting would fall primarily on pharmaceutical companies and CROs. The burden of improving real-world data, particularly reporting toxicities to the FAERS database and pharmaceutical companies, would fall largely on healthcare providers and pharmaceutical companies. Nevertheless, all stakeholders, including patients, medical organizations, and data scientists, can and should participate in strengthening clinical trial and real-world data about irAEs (figure 3).

In an effort to improve real-world data, in collaboration with irAE experts, Project Data Sphere has initiated an effort to create a multi-institutional registry for irAEs that will serve as an open-access repository for deidentified, 


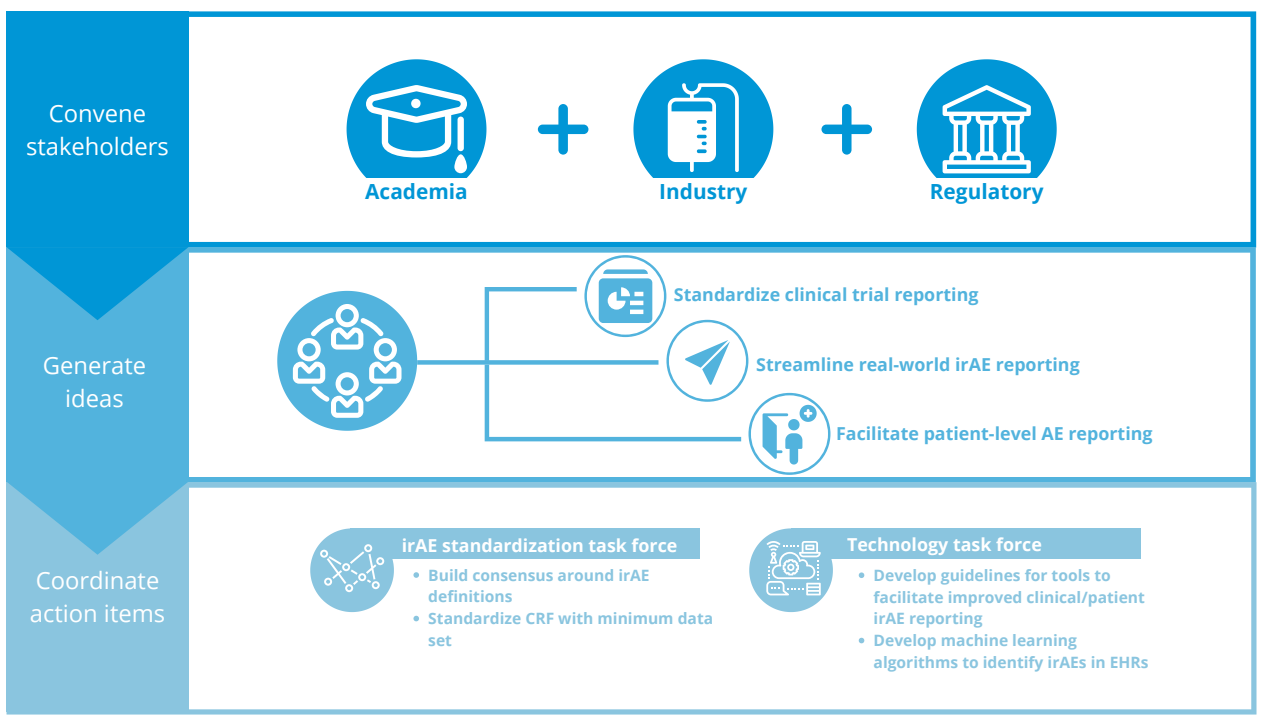

Figure 3 Stakeholders in multiple sectors, including academic, industry, and regulatory agencies, could help strengthen immune-related adverse event (irAE) data by convening task forces to standardize irAE definitions and reporting in clinical trials and develop technological tools to support real-world irAE reporting. AE, adverse event; CRF, case report forms; EHR, electronic health records.

patient-level information. ${ }^{13}$ The first phase of the registry will collect neurotoxicity data and is anticipated to be available publicly in 2 years. To establish the framework for the neurotoxicity registry, a set of standardized disease definitions and diagnostic workups for neurological irAEs, as well as a severity grading scale, were developed by a panel of neurologists, oncologists, and irAE specialists as described in this edition. This was the first proof of principle for such disease definitions and there are plans to expand to additional organ systems such as dermatology.

Although this article focuses on the role of researchers and clinicians in standardizing $\mathrm{AE}$ documentation in clinical trials and expanding real-world AE reporting, the part that patients play cannot be understated. Most critically, patients volunteer to participate in ICI clinical trials. In the context of both clinical trials and real-world settings, it is critical for patients to be vigilant about thoroughly reporting any suspected AEs to their medical team, even though they may be reluctant to discuss AEs out of fear that clinicians will discontinue their therapy. In this context, it is important for clinicians to discuss with their patients the importance of reporting possible toxicities as early as possible to initiate proper evaluation and treatment and mitigate their severity. To support patient reporting of AEs, pharmaceutical and data analytics companies are developing software applications, such as ApricityRx, to help patients learn about and report both low-grade and severe AEs. ${ }^{36}$ Ideally such software would have the capability to automatically fill in patient information and thus help patients submit toxicity data to drug sponsors and FAERS.

The most powerful messages about the need to improve our understanding of irAEs come from patients. The wife of a patient who received an ICI for advanced melanoma in early 2016 spoke at the FDA-PDS symposium. The ICI proved highly effective against the disease but caused multiple organ toxicities, including pneumonitis and colitis that, despite every effort by his clinical team, claimed his life 6 months after his diagnosis. In a telling story, his wife emphasized how grateful their family was that he was able to receive ICI combination therapy when no other treatment options were available, and that they do not regret their treatment decision. However, she urged pharmaceutical companies, clinicians, and researchers do all they can to share patient data to advance the science and make ICI therapy as safe as possible. The authors hope this article will advance our response to her plea. We owe it to all patients who are candidates for ICI therapy and their families.

\section{Summary}

This article draws attention to the major gaps in the collection and sharing of irAE data in the context of clinical trials and the real-world setting, and details certain areas where important progress can be made. A particular focus is placed on the standardization of $\mathrm{AE} / \mathrm{irAE}$ documentation in clinical trials and expanding the voluntary efforts of providers and patients to report toxicities in the postmarketing setting.

The overall benefits of responding to the calls of action presented here, including cooperative efforts to improve collection and analysis of irAE data, will require the work of multiple stakeholders, but could have lasting positive impact on the lives of numerous patients being treated with ICIs. It would lead to critical advances in the ability to study and manage irAEs, both in ICI monotherapy settings and in the growing applications of ICI combination therapy. It is likely that the use of ICIs, particularly in the context of combination therapy, will only continue to rise. 


\section{Author affiliations}

${ }^{1}$ Division of Hematology and Oncology, Massachusetts General Hospital, Harvard Medical School, Boston, Massachusetts, USA

${ }^{2}$ Office of Oncologic Diseases, US Food and Drug Administration, Silver Spring, Florida, USA

${ }^{3}$ Program Director, Project Data Sphere, Cary, North Carolina, USA

${ }^{4}$ President, Project Data Sphere, Cary, North Carolina, USA

${ }^{5}$ Global Head of Portfolio Strategy \& Innovation, Merck KGaA, Darmstadt, Germany

${ }^{6}$ Former Chief Medical Officer, Pfizer Inc, New York, New York, USA

${ }^{7}$ Chief Executive Officer, ASCO CancerLinQ LLC, Alexandria, Virginia, USA

${ }^{8}$ Division of Neuromuscular Disorders, Department of Neurology, Massachusetts

General Hospital, Harvard Medical School, Boston, Massachusetts, USA

${ }^{9}$ Division of Gastroenterology, Department of Medicine, Massachusetts General

Hospital, Harvard Medical School, Boston, Massachusetts, USA

${ }^{10}$ Division of Palliative Care, Department of Medicine, Massachusetts General Hospital, Harvard Medical School, Boston, Massachusetts, USA

${ }^{11}$ Division of Nephrology, Department of Medicine, Massachusetts General Hospital, Harvard Medical School, Boston, Massachusetts, USA

${ }^{12}$ Center for Immunology and Inflammatory Diseases and Center for Cancer Research, Department of Medicine, Massachusetts General Hospital, Harvard Medical School, Boston, Massachusetts, USA

${ }^{13}$ Division of Hematology/Oncology, Vanderbilt University Medical Center, Nashville, Tennessee, USA

${ }^{14}$ Center for Immuno-Oncology and Gastroenterology Cancer Center, Dana-Farber Cancer Institute, Boston, USA

${ }^{15}$ Cancer Therapy Evaluation Program, Division of Cancer Treatment and Diagnosis, National Cancer Institute, Bethesda, Maryland, USA

Twitter Mace Rothenberg @MaceRothenberg and 0sama Rahma @0samaRahma2 Contributors All authors contributed to the conception and writing of this Call-ToAction.

Funding Medical writing support was provided by Carina Storrs, Ph.D., independent science writer, and was funded by Project Data Sphere. Figures were prepared by Johnathan Rine, MSPH, MBA, of Project Data Sphere. We acknowledge Laurie Stockton, formerly of PDS who was the project manager for FDA-PDS Symposium VII on irAE. We would like to thank Sefanit Tesayne for being a tireless patient advocate for the improvement of therapeutics for patients with immunotherapy-related toxicity. LZ postdoctoral fellowship is supported by the Spanish Society of Medical Oncology Grant.

Competing interests KLR receives support from Teladoc and Project Data Sphere. MR owns shares of Pfizer Inc. ACG has served on advisory boards/consulted with Alexion, RA Pharma (UCB), and Momenta (Jansen). She also receives support from Project Data Sphere. MD has received support from Novartis, Eli Lilly, Moderna, Tillotts Pharma, ORIC Pharmaceuticals, and Partner Therapeutics. He is engaged with Moderna and Neoleukin Therapeutics. LZ has received support from Merck US DBJ has received support from BMS and Incyte and serves on advisory boards for Array Biopharma, BMS, Catalyst, lovance, Jansen, Merck, Novartis, and Oncosec. OR has received support from Merck, Celgene, Five Prime, GSK, Bayer, Roche, Puretech, Imvacx, and Sobi, has an immunotherapy patent pending, and serves as CMO of Outcomes4me.

\section{Patient consent for publication Not required.}

Provenance and peer review Not commissioned; externally peer reviewed.

Open access This is an open access article distributed in accordance with the Creative Commons Attribution Non Commercial (CC BY-NC 4.0) license, which permits others to distribute, remix, adapt, build upon this work non-commercially, and license their derivative works on different terms, provided the original work is properly cited, appropriate credit is given, any changes made indicated, and the use is non-commercial. See http://creativecommons.org/licenses/by-nc/4.0/.

\section{ORCID iDs}

Amanda C Guidon http://orcid.org/0000-0003-0843-2935

Michael Dougan http://orcid.org/0000-0001-9266-2009

Elad Sharon http://orcid.org/0000-0002-0044-9719

\section{REFERENCES}

1 Sise ME, Seethapathy H, Reynolds KL. Diagnosis and management of immune checkpoint inhibitor-associated renal toxicity: illustrative case and review. Oncologist 2019;24:735-42.

2 Haslam A, Prasad V. Estimation of the percentage of US patients with cancer who are eligible for and respond to checkpoint inhibitor immunotherapy drugs. JAMA Netw Open 2019;2:e192535.

3 Brahmer JR, Lacchetti C, Schneider BJ, et al. Management of immune-related adverse events in patients treated with immune checkpoint inhibitor therapy: American Society of clinical oncology clinical practice guideline. JCO 2018;36:1714-68.

4 Neilan TG, Rothenberg ML, Amiri-Kordestani L, et al. Myocarditis associated with immune checkpoint inhibitors: an expert consensus on data gaps and a call to action. Oncologist 2018;23:874-8.

5 Larkin J, Chiarion-Sileni V, Gonzalez R, et al. Five-Year survival with combined nivolumab and ipilimumab in advanced melanoma. N Engl $J$ Med 2019;381:1535-46.

6 Xu C, Chen Y-P, Du X-J, et al. Comparative safety of immune checkpoint inhibitors in cancer: systematic review and network metaanalysis. BMJ 2018;7:k4226.

7 Safa H, Johnson DH, Trinh VA, et al. Immune checkpoint inhibitor related myasthenia gravis: single center experience and systematic review of the literature. J Immunother Cancer 2019;7:319.

8 Reynolds KL, Sullivan RJ, Fintelmann FJ. Case 9-2020: a 64-yearold man with shortness of breath, cough, and hypoxemia. Cabot rC, Rosenberg ES, Pierce VM, et al., EDS. N Engl J Med 2020;382:1150-9.

9 Tang J, Shalabi A, Hubbard-Lucey VM. Comprehensive analysis of the clinical immuno-oncology landscape. Ann Oncol 2018;29:84-91.

10 Wang DY, Ye F, Zhao S, et al. Incidence of immune checkpoint inhibitor-related colitis in solid tumor patients: a systematic review and meta-analysis. Oncoimmunology 2017;6:e1344805.

11 Zhai Y, Ye X, Hu F, et al. Endocrine toxicity of immune checkpoint inhibitors: a real-world study Leveraging US food and drug administration adverse events reporting system. $J$ Immunother Cancer 2019;7:286.

12 Seethapathy H, Zhao S, Chute DF, et al. The incidence, causes, and risk factors of acute kidney injury in patients receiving immune checkpoint inhibitors. Clin J Am Soc Nephrol 2019;14:1692-700.

13 Project Data Sphere. Immune-Related adverse events. Available: https://www.projectdatasphere.org/research/programs/immunerelated-adverse-events [Accessed 26 Aug 2020].

14 Amiri-Kordestani L, Moslehi J, Cheng J, et al. Cardiovascular adverse events in immune checkpoint inhibitor clinical trials: a U.S. food and drug administration pooled analysis. JCO 2018;36:3009.

15 Xing P, Zhang F, Wang G, et al. Incidence rates of immune-related adverse events and their correlation with response in advanced solid tumours treated with NIVO or NIVO+IPI: a systematic review and meta-analysis. J Immunother Cancer 2019;7:341.

16 Stamatouli AM, Quandt Z, Perdigoto AL, et al. Collateral damage: insulin-dependent diabetes induced with checkpoint inhibitors. Diabetes 2018;67:1471-80.

17 Alliance for Clinical Trials in Oncology. Alliance to establish national Biorepository to advance study of immune-related side effects. Available: https://www.allianceforclinicaltrialsinoncology.org/main/ public/standard.xhtml?path=/Public/News-Alliance-BiobankJan2020 [Accessed 26 Aug 2020].

18 Dubin K, Callahan MK, Ren B, et al. Intestinal microbiome analyses identify melanoma patients at risk for checkpoint-blockade-induced colitis. Nat Commun 2016;7:10391.

19 Jing Y, Liu J, Ye Y, et al. Multi-Omics prediction of immune-related adverse events during checkpoint immunotherapy. Nat Commun 2020;11:4946.

20 Wargo J, Andrews M, Duong C. Gut microbiota signatures are associated with toxicity to combined CTLA- 4 and PD-1 blockade. Research Square 2020.

21 Thompson JA, Schneider BJ, Brahmer J, et al. Management of Immunotherapy-Related toxicities, version 1.2019, NCCN clinical practice guidelines in oncology. J Natl Compr Cancer Netw 2019;17:255-89.

22 Puzanov I, Diab A, Abdallah K, et al. Managing toxicities associated with immune checkpoint inhibitors: consensus recommendations from the Society for immunotherapy of cancer (SITC) toxicity management Working group. J Immunother Cancer 2017;5:95.

23 Haanen JBAG, Carbonnel F, Robert C, et al. Management of toxicities from immunotherapy: ESMO clinical practice guidelines for diagnosis, treatment and follow-up. Ann Oncol 2017;28:iv119-42.

24 Yu Y, Ruddy KJ, Tsuji S, et al. Coverage evaluation of CTCAE for capturing the immune-related adverse events Leveraging text mining technologies. AMIA Jt Summits Trans/ Sci Proc 2019;2019:771-8. 
25 Johnson DB, Reynolds KL, Sullivan RJ, et al. Immune checkpoint inhibitor toxicities: systems-based approaches to improve patient care and research. Lancet Oncol 2020;21:e398-404.

26 Qin S, Xu L, Yi M, et al. Novel immune checkpoint targets: moving beyond PD-1 and CTLA-4. Mol Cancer 2019;18:155.

27 Raschi E, Gatti M, Gelsomino F, et al. Lessons to be learnt from realworld studies on immune-related adverse events with checkpoint inhibitors: a clinical perspective from pharmacovigilance. Target Oncol 2020;15:449-66.

28 U.S. Food and Drug Administration. MedWatch online reporting form. Available: https://www.accessdata.fda.gov/scripts/medwatch/index. cfm [Accessed 7 May 2021].

29 U.S. Food and Drug Administration. Potential signals of serious Risks/New safety information identified from the FDA adverse event reporting system (FAERS). Available: https://www.fda.gov/drugs/ questions-and-answers-fdas-adverse-event-reporting-system-faers/ potential-signals-serious-risksnew-safety-information-identified-fdaadverse-event-reporting-system [Accessed 25 Aug 2020].

$30 \mathrm{Ji} \mathrm{H}-\mathrm{H}$, Tang X-W, Dong Z, et al. Adverse event profiles of anti-CTLA-4 and anti-PD-1 monoclonal antibodies alone or in combination: analysis of spontaneous reports submitted to FAERS. Clin Drug Investig 2019;39:319-30.
31 Johnson DB, Manouchehri A, Haugh AM, et al. Neurologic toxicity associated with immune checkpoint inhibitors: a pharmacovigilance study. J Immunother Cancer 2019;7:134.

32 Myint Z, Qasrawi A, O'Neal R, et al. Immune-Related adverse events for different age groups using the FDA adverse event reporting system. JCO 2019;37:e14256.

33 Spillane S, Baxi S, Torres AZ, et al. Organ dysfunction in patients with advanced melanoma treated with immune checkpoint inhibitors. Oncologist 2020;25:e1753-62.

34 U.S. Food and Drug Administration. An update to the FDA adverse event reporting system (FAERS) public Dashboard. Available: https://www.fda.gov/media/122827/download [Accessed 7 May 2021].

$35 \mathrm{Yu}$ Y, Ruddy KJ, Wen A, et al. Integrating electronic health record data into the ADEpedia-on-OHDSI platform for improved signal detection: a case study of immune-related adverse events. AMIA Jt Summits Trans/ Sci Proc 2020;2020:710-9.

36 Campbell MT, Zhang T, Chin L, et al. ApricityRx companion digital therapeutic for evidence-based mitigation and phenotype-linked molecular characterization of irAEs in patients receiving immune checkpoint therapy (ICT). JCO 2020;38:TPS2089. 